\title{
The Fischer-Cliford Matrices and Character Table of the Group $2^{5}: G L(4,2)$
}

Rauhi I. Elkhatib

\author{
Dept. of Mathematics, Faculty of Applied Science, Thamar University, Yemen
}

*Corresponding Author: Rauhi I. Elkhatib, Dept. of Mathematics, Faculty of Applied Science, Thamar University, Yemen

Abstract: The purpose of this paper is constructing the Fischer-Clifford matrices and the character tables for the group $\mathbf{2}^{5}: \mathbf{G L}(\mathbf{4}, \mathbf{2})$.

Keywords: linear groups, group extensions, character table, Clifford theory, inertia groups, Fischer-Clifford matrix.

\section{INTRODUCTION}

The theory of Clifford-Fischer matrices, which is based on Clifford Theory [1] which was developed by B. Fischer [2]. Let $\bar{G}=2^{5}: G L(4,2)$ be the split extension of $N=2^{5}$ by $G L(4,2)$ where $\mathrm{N}$ is the vector space of dimension 5 over $\mathrm{GF}(2)$ on which $\mathrm{G}$ acts naturally. The aim of this paper is to construct the character table of $\bar{G}$ by using the technique of Fischer-Clifford matrix $M(g)$ for each class representative $\mathrm{g}$ of $\mathrm{G}$ and the character tables of the inertia factor groups $H_{i}$ of the inertia groups $\overline{H_{i}}=2^{5}: H_{i}$. we use the properties of the Fischer-Clifford matrices discussed in ( [3], [4],[5], [6], [7], [8], [9], [10], [11], [12], [13], [14], [15]) to compute entries of these matrices. The Fischer-Clifford matrix $M(g)$ will be portioned row-wise into blocks, where each block corresponds to an inertia group $\overline{H_{i}}$. Now using the columns of character table of the inertia factor $H_{i}$ of $\overline{H_{i}}$ which correspond to classes of $H_{i}$ which fuse to the class [g] in $\mathrm{G}$ and multiply these columns by the rows of the FischerClifford matrix $M(g)$ that correspond to $\overline{H_{i}}$. Thus, we construct the portion of the character table of $\bar{G}$ which is in the block corresponding to $\overline{H_{i}}$ for the classes of $\bar{G}$ that come from the coset $\mathrm{Ng}$. For more information about this technique see ( [3], [4],[5], [6], [7], [8], [9], [10], [11], [12], [13], [14], [15]) . The character table of $\bar{G}$ will be divided row-wise into blocks where each block corresponding to an inertia group $\overline{H_{i}}=N: H_{i}$. The computations have been carried out with the aid of Maxima [16], MAGMA [17] and GAP [18], Finally we will follow the notion of Atlas [19].

\section{THEORY OF FISCHER-CLIFFORD MATRICES}

Let $\bar{G}=N: G$ be a split extension of $\mathrm{N}$ by $\mathrm{G}$. Then for $\theta \in \operatorname{Irr}(N)$, we define $\bar{H}=\left\{x \in \bar{G}: \theta^{x}=\right.$ $\theta=I G \theta$ and $H=x \in G: \theta g=\theta=I G \theta$ where $I G \theta$ is the stabilizer of $\theta$ in the action of $G$ on $\operatorname{Irr}(\mathrm{N})$, we have that $I_{\bar{G}}(\theta)$ is a subgroup of $\bar{G}$ and $\mathrm{N}$ is normal subgroup in $I_{\bar{G}}(\theta)$. Also $\left[\bar{G}: I_{\bar{G}}(\theta)\right]$ is the size of the orbit containing $\theta$. Then it can be shown that $\bar{H}=N: H$, where $\bar{H}$ is the inertia group of $\theta$ in $\bar{G}$. The inertia factor $\bar{H} / N \cong H$ can be regarded as the inertia group of $\theta$ in the factor group $\bar{G} / N \cong G$. Define $\theta^{g}$ by $\theta^{g}(n)=\theta\left(g n g^{-1}\right)$ for $g \in \bar{G}, n \in N, \theta^{g} \in \operatorname{Irr}(N)$. We say that $\theta$ is extendible to $\bar{H}$ if there exists $\varphi \in \operatorname{Irr}(\bar{H})$ such that $\downarrow_{N}=\theta$. If $\theta$ is extendible to $\bar{H}$, then by Gallagher [20], we have $\left\{\alpha: \alpha \in \operatorname{Irr}(\bar{H}),\left\langle\alpha \downarrow_{N}, \theta>\neq 0\right\}=\{\beta \varphi: \beta \in \operatorname{Irr}(\bar{H} / N)\}\right.$. Let $\bar{G}$ has the property that every irreducible character of $\mathrm{N}$ can be extended to its inertia group. Now let $\theta_{1}=1_{N}, \theta_{2}, \ldots, \theta_{t}$ be representatives of the orbits of $\bar{G}$ on $\operatorname{Irr}(\mathrm{N}), \overline{H_{i}}=I_{\bar{G}}\left(\varphi_{i}\right), 1 \leq i \leq t, \varphi_{i} \in \operatorname{Irr}\left(\overline{H_{i}}\right)$ be an extension of $\theta_{i}$ to $\overline{H_{i}}$ and $\beta \in \operatorname{Irr}\left(\overline{H_{i}}\right)$ such that $N \subseteq \operatorname{Ker}(\beta)$. Then it can be shown that $\operatorname{Irr}(\bar{G})=\bigcup_{i=1}^{t}\left\{\left(\beta \varphi_{i}\right)^{\bar{G}}: \beta \in \operatorname{Irr}\left(\overline{H_{i}}\right), N \subseteq \operatorname{Ker}(\beta)\right\}=\bigcup_{i=1}^{t}\left\{\left(\beta \varphi_{i}\right)^{\bar{G}}: \beta \in\left(\overline{H_{i}} / N\right)\right\}$ 
Hence the irreducible characters of $\bar{G}$ will be divided into blocks, where each block corresponds to an inertia group $\overline{H_{i}}$. Let $\overline{H_{i}}$ be the inertia factor group and $\varphi_{i}$ be an extension of $\theta_{i}$ to $\overline{H_{i}}$. Take $\theta_{1}=1_{N}$ as the identity character of $\mathrm{N}$, then $\overline{H_{1}}=\bar{G}$ and $H_{1} \cong G$. Let $X(g)=\left\{x_{1}, x_{2}, \ldots, x_{c(g)}\right\}$ be a set of representatives of the conjugacy classes of $\bar{G}$ from the coset $N \bar{g}$ whose images under the natural homomorphism $\bar{G} \rightarrow G$ are in $[\mathrm{g}]$ and we take $x_{1}=\bar{g}$. We define

$$
R(g)=\left\{\left(i, y_{k}\right): 1 \leq i \leq t, H_{i} \cap[g] \neq 0,1 \leq k \leq r\right\}
$$

and we note that $\mathrm{y}_{\mathrm{k}}$ runs over representatives of the conjugacy classes of elements of $\mathrm{H}_{\mathrm{i}}$ which fuse into $[\mathrm{g}]$ in G. Then we define the Fischer-Clifford matrix $\mathrm{M}(\mathrm{g})$ by $M(g)=\left(a_{\left(i, y_{k}\right)}^{j}\right)$, where $a_{\left(i, y_{k}\right)}^{j}=$ $\sum_{l}^{t} \frac{\left|C_{\bar{G}}\left(x_{j}\right)\right|}{\left|C\left(y_{l k}\right)\right|} \varphi_{i}\left(y_{l k}\right)$ with columns indexed by $\mathrm{X}(\mathrm{g})$ and rows indexed by $\mathrm{R}(\mathrm{g})$ and where $\sum_{l}^{t}$ is the summation over all $l$ for which $y_{l k} \sim x_{j}$ in $\bar{G}$. Then the partial character table of $\bar{G}$ on the classes $\left\{x_{1}, x_{2}, \ldots, x_{c(g)}\right\}$ is given by $\left[\begin{array}{c}C_{1}(g) M_{1}(g) \\ C_{2}(g) M_{2}(g) \\ \vdots \\ C_{t}(g) M_{t}(g)\end{array}\right]$ where the Fischer-Clifford matrix $M(g)=\left[\begin{array}{c}M_{1}(g) \\ M_{2}(g) \\ \vdots \\ M_{t}(g)\end{array}\right]$ is divided into blocks $M(g)$ with each block corresponding to an inertia group $\overline{H_{i}}$ and $C_{i}(g)$ is the partial character table of $H_{i}$ consisting of the columns corresponding to the classes that fuse into [g] in G. We can also observe that the number of irreducible characters of $\bar{G}$ is the sum of the number of irreducible characters of the inertia factors $H_{i}{ }^{\prime} s$. The group $\bar{G}=2^{5}: G L(4,2)$ is a split extension with $2^{5}$ abelian and therefore by Mackey's theorem (see [8] - Theorem 4.1.12), we have each irreducible character of $2^{5}$ can be extended to its inertia group in $\bar{G}$. Hence by the above theoretical outline we can fully determine the character table of $\bar{G}=2^{5}: G L(4,2)$.

\section{THE CONJUGACY CLASSES OF $\overline{\mathrm{G}}=2^{5}: \operatorname{GL}(4,2)$}

In this section, we will use the method of coset analysis to determine the conjugacy classes of $\bar{G}=2^{5}: G L(4,2)$. We refer the reader to ( [3], [4],[5], [6], [7], [8], [9], [10], [11], [12], [13], [14], [15]) for full details and background material regarding the method of coset analysis. Most of the information, which involved the conjugacy classes and permutation characters, were obtained by using direct computations in GAP [18] and MAGMA [17]. The general linear group $G L(4,2)$ of order $=20160$ is a subgroup of the general linear group $G L(5,2)$. By MAGMA [17], We can generate the group GL $(4,2)$ by the two $5 \times 5$ matrices:

$a=\left[\begin{array}{lllll}1 & 0 & 0 & 0 & 0 \\ 0 & 1 & 1 & 0 & 0 \\ 0 & 0 & 1 & 0 & 0 \\ 0 & 0 & 0 & 1 & 0 \\ 0 & 0 & 0 & 0 & 1\end{array}\right], \quad b=\left[\begin{array}{lllll}1 & 0 & 0 & 0 & 0 \\ 0 & 0 & 0 & 0 & 1 \\ 0 & 1 & 0 & 0 & 0 \\ 0 & 0 & 1 & 0 & 0 \\ 0 & 0 & 0 & 1 & 0\end{array}\right]$

We construct the conjugacy class representative of $\mathrm{G}$ in terms of $5 \times 5$ matrices over GF(2) by GAP [18]. The GL $(4,2)$ has 14 conjugacy classes and under the action of GL $(4,2)$ on $2^{5}$, we obtain four orbits of lengths $1,15,1$ and 15. The point stabilizers for orbits of lengths 1, 15, 1 and 15 are the subgroups GL $(4,2), 2^{3}: \operatorname{PSL}(3,2), A_{8}$ and $2^{3}: \operatorname{PSL}(3,2)$ of indices $1,15,1$ and 15 , respectively in GL $(4,2)$. Let $X\left(G L(4,2) \mid 2^{5}\right)$ be the permutation character of GL(4,2) on $2^{5}$. The values of $\chi\left(G L(4,2) \mid 2^{5}\right)$ on different classes of GL $(4,2)$ determine the number of $k$ of fixed points of each conjugacy class of $\mathrm{GL}(4,2)$ in $2^{5}$. These values of the $k^{\prime} s$ will help us to calculate the conjugacy classes of $2^{5}: G L(4,2)$ which are listed in Table 1 . Consequentially, having obtained the values of the $k^{\prime} s$ for the various classes of $\mathrm{G}$, we then need to calculate the values of $f_{i}{ }^{\prime} s$ corresponding to the various 's, where $f_{i}^{\prime} s$ are the number of orbits $Q_{i}$ 's for $1 \leq i \leq k$, that fuse together under the action 
of $C_{G}(g)$ to form one orbit $\Delta_{j}$. For this purpose, we used Programme A [8]. For a class representative $d g \in \bar{G}$, where $d \in 2^{5}, g \in G L(4,2)$ and $o(g)=m$, by Theorem 3.3.10 in [15] we have

$$
o(d g)= \begin{cases}m & \text { if } w=1_{N} \\ 2 m & \text { otherwise }\end{cases}
$$

To calculate the orders of the class representative $d g \in \bar{G}$, we used Programme B [8]. If $o(g)=m$ and $w=1_{N}$ then $o(d g)=2 m$ otherwise if $\neq 1_{N}$, then $o(d g)=2 m$. To each class of $\bar{G}$, we have attached some weight $m_{i j}$ which will be used later in computing the Fischer matrices of the extension. These weights are computed by the formula

$$
m_{i j}=\left[N_{\bar{G}}\left(N \bar{g}_{i}\right): C_{\bar{G}}\left(g_{i j}\right)\right]=|N| \frac{\left|C_{G}\left(g_{i}\right)\right|}{\left|C_{\bar{G}}\left(g_{i j}\right)\right|}
$$

Thus, we obtained 50 conjugacy classes for the group $\bar{G}=2^{5}: G L(4,2)$ and we list these result about the conjugacy classes of $\bar{G}$ in Table 1 .

\begin{tabular}{|c|c|c|c|c|c|c|c|c|}
\hline$g_{i} \in G$ & $k_{i}$ & $f_{i}$ & $m_{i, j}$ & $d_{i}$ & $w$ & {$[x]_{\bar{G}}$} & $\left|[x]_{\bar{G}}\right|$ & $\left|C_{\bar{G}}(x)\right|$ \\
\hline \multirow[t]{4}{*}{$1 \mathrm{~A}$} & \multirow[t]{4}{*}{32} & 1 & 1 & $(0,0,0,0,0)$ & $(0,0,0,0,0)$ & $g_{1,1}=1 a$ & 1 & 645120 \\
\hline & & 1 & 1 & $(1,0,0,0,0)$ & $(1,0,0,0,0)$ & $g_{1,2}=2 a$ & 1 & 645120 \\
\hline & & 15 & 15 & $(0,0,0,0,1)$ & $(0,0,0,0,1)$ & $g_{, 13}=2 b$ & 15 & 43008 \\
\hline & & 15 & 15 & $(1,0,0,0,1)$ & $(1,0,0,0,1)$ & $g_{1,4}=2 c$ & 15 & 43008 \\
\hline \multirow[t]{2}{*}{$3 \mathrm{~A}$} & \multirow[t]{2}{*}{2} & 1 & 16 & $(0,0,0,0,0)$ & $(0,0,0,0,0)$ & $g_{2,1}=3 a$ & 1792 & 360 \\
\hline & & 1 & 16 & $(1,0,0,0,0)$ & $(1,0,0,0,0)$ & $g_{2,2}=6 a$ & 1792 & 360 \\
\hline \multirow[t]{2}{*}{$5 \mathrm{~A}$} & \multirow[t]{2}{*}{2} & 1 & 16 & $(0,0,0,0,0)$ & $(0,0,0,0,0)$ & $g_{3,1}=5 a$ & 21504 & 30 \\
\hline & & 1 & 16 & $(1,0,0,0,0)$ & $(1,0,0,0,0)$ & $g_{3,2}=10 a$ & 21504 & 30 \\
\hline \multirow[t]{2}{*}{$15 \mathrm{~A}$} & \multirow[t]{2}{*}{2} & 1 & 16 & $(0,0,0,0,0)$ & $(0,0,0,0,0)$ & $g_{4,1}=15 a$ & 21504 & 30 \\
\hline & & 1 & 16 & $(1,0,0,0,0)$ & $(1,0,0,0,0)$ & $g_{4,2}=30 a$ & 21504 & 30 \\
\hline \multirow[t]{2}{*}{$15 \mathrm{~B}$} & \multirow[t]{2}{*}{2} & 1 & 16 & $(0,0,0,0,0)$ & $(0,0,0,0,0)$ & $g_{5,1}=15 b$ & 21504 & 30 \\
\hline & & 1 & 16 & $(1,0,0,0,0)$ & $(1,0,0,0,0)$ & $g_{5,2}=30 b$ & 21504 & 30 \\
\hline \multirow[t]{4}{*}{$7 \mathrm{~A}$} & \multirow[t]{4}{*}{4} & 1 & 8 & $(0,0,0,0,0)$ & $(0,0,0,0,0)$ & $g_{6,1}=7 a$ & 23040 & 28 \\
\hline & & 1 & 8 & $(0,0,0,1,0)$ & $(0,1,1,0,0)$ & $g_{6,2}=14 a$ & 23040 & 28 \\
\hline & & 1 & 8 & $(1,0,0,0,0)$ & $(1,0,0,0,0)$ & $g_{6,3}=14 b$ & 23040 & 28 \\
\hline & & 1 & 8 & $(1,0,0,1,0)$ & $(1,1,1,0,0)$ & $g_{6,4}=14 c$ & 23040 & 28 \\
\hline \multirow[t]{4}{*}{$7 \mathrm{~B}$} & \multirow[t]{4}{*}{4} & 1 & 8 & $(0,0,0,0,0)$ & $(0,0,0,0,0)$ & $g_{7,1}=7 b$ & 23040 & 28 \\
\hline & & 1 & 8 & $(0,0,0,1,0)$ & $(0,1,1,0,0)$ & $g_{7,2}=14 d$ & 23040 & 28 \\
\hline & & 1 & 8 & $(1,0,0,0,0)$ & $(1,0,0,0,0)$ & $g_{7,3}=14 e$ & 23040 & 28 \\
\hline & & 1 & 8 & $(1,0,0,1,0)$ & $(1,1,1,0,0)$ & $g_{7,4}=14 f$ & 23040 & 28 \\
\hline \multirow[t]{4}{*}{$2 \mathrm{~A}$} & \multirow[t]{4}{*}{8} & 1 & 4 & $(0,0,0,0,0)$ & $(0,0,0,0,0)$ & $g_{8,1}=2 d$ & 840 & 768 \\
\hline & & 1 & 4 & $(1,0,0,0,0)$ & $(0,0,0,0,0)$ & $g_{8,2}=2 e$ & 840 & 768 \\
\hline & & 3 & 12 & $(0,0,0,0,1)$ & $(0,1,0,1,0)$ & $g_{8,3}=4 a$ & 2520 & 256 \\
\hline & & 3 & 12 & $(1,0,0,0,1)$ & $(0,1,0,1,0)$ & $g_{8,4}=4 b$ & 2520 & 256 \\
\hline \multirow[t]{4}{*}{$4 \mathrm{~A}$} & \multirow[t]{4}{*}{4} & 1 & 8 & $(0,0,0,0,0)$ & $(0,0,0,0,0)$ & $g_{9,1}=4 c$ & 20160 & 32 \\
\hline & & 1 & 8 & $(0,0,0,0,1)$ & $(0,1,1,0,0)$ & $g_{9,2}=8 a$ & 20160 & 32 \\
\hline & & 1 & 8 & $(1,0,0,0,0)$ & $(0,0,0,0,0)$ & $g_{9,3}=4 d$ & 20160 & 32 \\
\hline & & 1 & 8 & $(1,0,0,0,1)$ & $(0,1,1,0,0)$ & $g_{9,4}=8 b$ & 20160 & 32 \\
\hline \multirow[t]{6}{*}{ 2B } & \multirow[t]{6}{*}{16} & 1 & 2 & $(0,0,0,0,0)$ & $(0,0,0,0,0)$ & $g_{10,1}=2 f$ & 210 & 3072 \\
\hline & & 4 & 8 & $(0,0,0,0,1)$ & $(0,1,1,0,0)$ & $g_{10,2}=4 e$ & 840 & 768 \\
\hline & & 3 & 6 & $(0,0,0,1,0)$ & $(0,0,0,0,0)$ & $g_{10,3}=2 g$ & 630 & 1024 \\
\hline & & 1 & 2 & $(1,0,0,0,0)$ & $(0,0,0,0,0)$ & $g_{10,4}=2 h$ & 210 & 3072 \\
\hline & & 4 & 8 & $(1,0,0,0,1)$ & $(0,1,1,0,0)$ & $g_{10,5}=4 f$ & 840 & 768 \\
\hline & & 3 & 6 & $(1,0,0,1,0)$ & $(0,0,0,0,0)$ & $g_{10,6}=2 i$ & 630 & 1024 \\
\hline \multirow[t]{2}{*}{$3 \mathrm{~B}$} & \multirow[t]{2}{*}{8} & 1 & 4 & $(0,0,0,0,0)$ & $(0,0,0,0,0)$ & $g_{11,1}=3 b$ & 4480 & 144 \\
\hline & & 3 & 8 & $(0,0,0,0,1)$ & $(0,1,0,1,1)$ & $g_{11,2}=6 b$ & 13440 & 48 \\
\hline
\end{tabular}

Table 1. Conjugacy Classes of $\bar{G}=2^{5}: G L(4,2)$ 
The Fischer-Cliford Matrices and Character Table of the Group $2^{5}: G L(4,2)$

\begin{tabular}{|c|c|c|c|c|c|c|c|c|}
\hline \hline & & 1 & 4 & $(1,0,0,0,0)$ & $(1,0,0,0,0)$ & $g_{11,3}=6 c$ & 4480 & 144 \\
& & 3 & 8 & $(1,0,0,0,1)$ & $(1,1,0,1,1)$ & $g_{11,4}=6 d$ & 13440 & 48 \\
\hline 6A & 4 & 1 & 8 & $(0,0,0,0,0)$ & $(0,0,0,0,0)$ & $g_{12,1}=6 e$ & 26880 & 24 \\
& & 1 & 8 & $(0,0,0,0,1)$ & $(0,1,1,0,0)$ & $g_{12,2}=12 a$ & 26880 & 24 \\
& & 1 & 8 & $(1,0,0,0,0)$ & $(0,0,0,0,0)$ & $g_{12,3}=6 f$ & 26880 & 24 \\
& & 1 & 8 & $(1,0,0,0,1)$ & $(0,1,1,0,0)$ & $g_{12,4}=12 b$ & 26880 & 24 \\
\hline 4B & 8 & 1 & 4 & $(0,0,0,0,0)$ & $(0,0,0,0,0)$ & $g_{13,1}=4 g$ & 5040 & 128 \\
& & 2 & 8 & $(0,0,0,0,1)$ & $(0,0,0,0,0)$ & $g_{13,2}=4 h$ & 10080 & 64 \\
& & 1 & 4 & $(0,0,1,0,0)$ & $(0,0,0,0,0)$ & $g_{13,3}=4 i$ & 5040 & 128 \\
& & 1 & 4 & $(1,0,0,0,0)$ & $(0,0,0,0,0)$ & $g_{13,4}=4 j$ & 5040 & 128 \\
& & 2 & 8 & $(1,0,0,0,1)$ & $(0,0,0,0,0)$ & $g_{13,5}=4 k$ & 10080 & 64 \\
& & 1 & 4 & $(1,0,1,0,0)$ & $(0,0,0,0,0)$ & $g_{13,6}=4 l$ & 5040 & 128 \\
\hline 6b & 2 & 1 & 16 & $(0,0,0,0,0)$ & $(0,0,0,0,0)$ & $g_{14,1}=6 g$ & 26880 & 24 \\
& & & 16 & $(1,0,0,0,0)$ & $(0,0,0,0,0)$ & $g_{14,2}=6 h$ & 26880 & 24 \\
\hline
\end{tabular}

Thus, the group $2^{5}: G L(4,2)$ has 50 conjugacy classes.

\section{THE INERTIA FACTOR Group OF $\overline{\mathrm{G}}=2^{5}: \operatorname{GL}(4,2)$}

The action of $\mathrm{G}$ on $\mathrm{N}$ produce four orbits of lengths 1, 15, 1 and 15. Hence by Brauer'stheorem ( see Theorem 5.1.4 in[15]) $\mathrm{G}$ acts on $\operatorname{Irr}(\mathrm{N})$ with the same number of orbits. The lengths of the these orbits will be $1, r, s, p$ where $1+r+s+p=32$, with corresponding point stabilizers $H_{1}, H_{2}, H_{3}$ and $H_{4}$ as subgroups of $G$ such that $\left[G: H_{1}\right]=1,\left[G: H_{2}\right]=15,\left[G: H_{3}\right]=1$ and $\left[G: H_{4}\right]=15$. Considering the indices of these subgroups in $G$ and investigating the maximal and submaximal subgroups of $G$, we have the Group $G L(4,2)$ acting on $\operatorname{Irr}\left(2^{5}\right)$ produce four inertia factor groups:

(1) $H_{1}=G L(4,2)$ of index equal 1 ,

(2) $H_{2}=H_{4}=2^{3}: \operatorname{PSL}(3,2)$ of index equal 15 ,

(3) $H_{3}=A_{8}$ of index equal to 1 .

Using GAP [18], we can generate the group $H_{2}=H_{4}$ in terms of $5 \times 5$ matrices over GF(2) by the follows matrices:

$$
\begin{aligned}
& a_{1}=\left[\begin{array}{lllll}
1 & 0 & 0 & 0 & 0 \\
0 & 1 & 1 & 0 & 0 \\
0 & 0 & 1 & 0 & 0 \\
0 & 0 & 0 & 1 & 0 \\
0 & 0 & 0 & 0 & 1
\end{array}\right], a_{2}=\left[\begin{array}{lllll}
1 & 0 & 0 & 0 & 0 \\
0 & 1 & 0 & 0 & 0 \\
0 & 0 & 1 & 1 & 0 \\
0 & 0 & 0 & 1 & 0 \\
0 & 0 & 0 & 0 & 1
\end{array}\right], \\
& a_{3}=\left[\begin{array}{lllll}
1 & 0 & 0 & 0 & 0 \\
0 & 1 & 0 & 0 & 0 \\
0 & 0 & 1 & 0 & 0 \\
0 & 0 & 0 & 1 & 1 \\
0 & 0 & 0 & 0 & 1
\end{array}\right], a_{4}=\left[\begin{array}{lllll}
1 & 0 & 0 & 0 & 0 \\
0 & 1 & 0 & 0 & 0 \\
0 & 0 & 1 & 0 & 0 \\
0 & 1 & 0 & 1 & 1 \\
0 & 0 & 0 & 0 & 1
\end{array}\right]
\end{aligned}
$$

By using these generators in GAP [18], we obtained the conjugacy classes and the character tables for this group.

We can complete the fusion maps by using matrix conjugation into the group $(4,2)$. This fusion map is listed in table 2 .

Table 2. The Fusion of the group $H_{2}=H_{4}=2^{3}: \operatorname{PSL}(3,2)$ into the group $G=G L(4,2)$

\begin{tabular}{|lll|llc|lcc|}
\hline$\lceil g\rceil_{H_{2}}$ & $\Rightarrow$ & $\lceil g\rceil_{G}$ & $\lceil g\rceil_{H_{2}}$ & $\Rightarrow$ & $\lceil g\rceil_{G}$ & $\lceil g\rceil_{H_{2}}$ & $\Rightarrow$ & $\lceil g\rceil_{G}$ \\
\hline $1 \mathrm{a}$ & $\Rightarrow$ & $1 \mathrm{a}$ & $2 \mathrm{a}$ & $\Rightarrow$ & $2 \mathrm{~b}$ & $2 \mathrm{~b}$ & $\Rightarrow$ & $2 \mathrm{~b}$ \\
\hline $4 \mathrm{a}$ & $\Rightarrow$ & $4 \mathrm{~b}$ & $2 \mathrm{c}$ & $\Rightarrow$ & $2 \mathrm{a}$ & $3 \mathrm{a}$ & $\Rightarrow$ & $3 \mathrm{~b}$ \\
\hline $6 \mathrm{a}$ & $\Rightarrow 6 \mathrm{a}$ & & $4 \mathrm{~b}$ & $\Rightarrow$ & $4 \mathrm{~b}$ & $4 \mathrm{c}$ & $\Rightarrow$ & $4 \mathrm{a}$ \\
\hline $7 \mathrm{a}$ & $\Rightarrow$ & $7 \mathrm{a}$ & $7 \mathrm{~b}$ & $\Rightarrow$ & $7 \mathrm{~b}$ & & & \\
\hline
\end{tabular}


Also, by using GAP [18], we can generate the group $H_{3}=A_{8}$ in terms of $5 \times 5$ matrices over GF(2) by the follows matrices:

$$
b_{1}=\left[\begin{array}{lllll}
1 & 0 & 0 & 0 & 0 \\
0 & 1 & 1 & 0 & 0 \\
0 & 0 & 1 & 0 & 0 \\
0 & 0 & 0 & 1 & 0 \\
0 & 0 & 0 & 0 & 1
\end{array}\right], b_{2}=\left[\begin{array}{lllll}
1 & 0 & 0 & 0 & 0 \\
0 & 0 & 0 & 0 & 1 \\
0 & 1 & 0 & 0 & 0 \\
0 & 0 & 1 & 0 & 0 \\
0 & 0 & 0 & 1 & 0
\end{array}\right]
$$

By using these generators in GAP [18], we obtained the conjugacy classes and the character tables for this group.

We can complete the fusion maps by using matrix conjugation in the group $(4,2)$. This fusion map is listed in table 3.

Table 3. The Fusion of the group $H_{3}=A_{8}$ into the group $G=G L(4,2)$

\begin{tabular}{|lll|lll|lll|}
\hline$\lceil g\rceil_{H_{3}}$ & $\Rightarrow$ & $\lceil g\rceil_{G}$ & $\lceil g\rceil_{H_{3}}$ & $\Rightarrow$ & $\lceil g\rceil_{G}$ & $\lceil g\rceil_{H_{3}}$ & $\Rightarrow$ & $\lceil g\rceil_{G}$ \\
\hline $1 \mathrm{a}$ & $\Rightarrow$ & $1 \mathrm{a}$ & $2 \mathrm{a}$ & $\Rightarrow$ & $2 \mathrm{a}$ & $4 \mathrm{a}$ & $\Rightarrow$ & $4 \mathrm{a}$ \\
\hline $2 \mathrm{~b}$ & $\Rightarrow$ & $2 \mathrm{~b}$ & $3 \mathrm{a}$ & $\Rightarrow$ & $3 \mathrm{~b}$ & $6 \mathrm{a}$ & $\Rightarrow$ & $6 \mathrm{a}$ \\
\hline $3 \mathrm{~b}$ & $\Rightarrow$ & $3 \mathrm{a}$ & $5 \mathrm{a}$ & $\Rightarrow$ & $5 \mathrm{a}$ & $15 \mathrm{a}$ & $\Rightarrow$ & $15 \mathrm{~b}$ \\
\hline $15 \mathrm{~b}$ & $\Rightarrow$ & $15 \mathrm{a}$ & $7 \mathrm{a}$ & $\Rightarrow$ & $7 \mathrm{a}$ & $7 \mathrm{~b}$ & $\Rightarrow$ & $7 \mathrm{~b}$ \\
\hline $6 \mathrm{~b}$ & $\Rightarrow$ & $6 \mathrm{~b}$ & $4 \mathrm{~b}$ & $\Rightarrow$ & $4 \mathrm{~b}$ & & & \\
\hline
\end{tabular}

\section{THE FisCHER-CLIFFORD MATRICES OF $\overline{\mathrm{G}}=2^{5}$ : $\mathrm{GL}(4,2)$}

For each conjugacy class $[\mathrm{g}]$ of $\mathrm{G}$ with representative $g \in G$, we construct the corresponding FischerClifford matrix $M(g)$ of $\bar{G}=2^{5}: G L(4,2)$. We use the properties of the Fischer-Clifford matrices see ( [3], [4],[5], [6], [7], [8], [9], [10], [11], [12], [13], [14], [15]) together with fusions of $H_{2}$ into $H_{1}, H_{3}$ into $H_{1}$ and $H_{4}$ into $H_{1}$ (Table 2, Table 3) to compute the entries of these matrices and to construct an algebraic system of linear and non-linear equations with the help of Maxima [16], we can solve these system of equations and compute all the Fischer matrices of $\bar{G}$. The Fischer-Clifford matrix will be partitioned row-wise into blocks, where each block corresponding to an inertia group $\overline{H_{i}}$. We list the Fischer-Clifford matrices of $\bar{G}$ in Table 4:

Table 4. The Fischer-Clifford Matrices of $2^{5}: G L(4,2)$ :

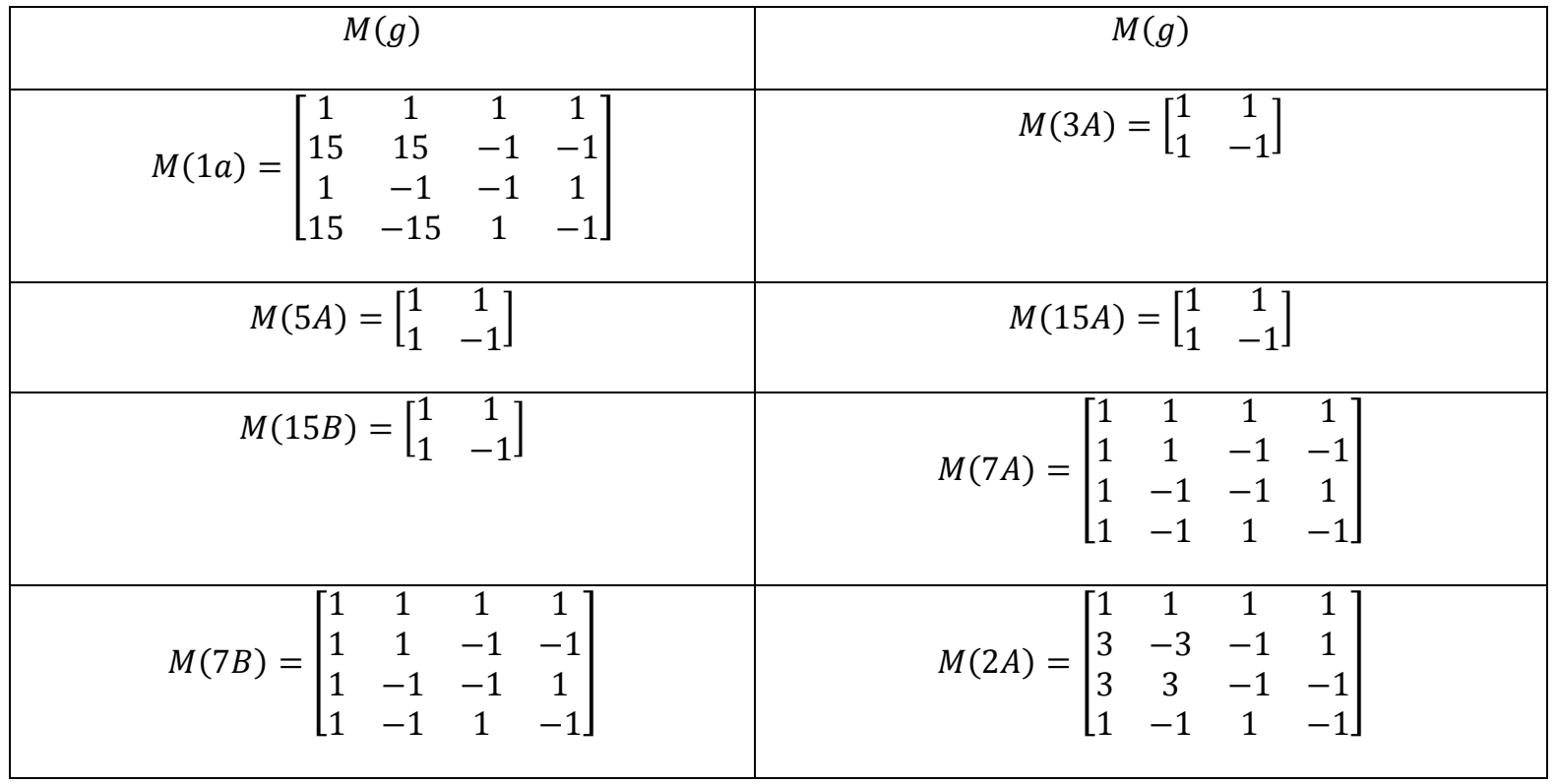


The Fischer-Cliford Matrices and Character Table of the Group $2^{5}: G L(4,2)$

\begin{tabular}{|c|c|c|}
\hline \hline$(4 A)=\left[\begin{array}{cccc}1 & 1 & 1 & 1 \\
1 & 1 & -1 & -1 \\
1 & -1 & -1 & 1 \\
1 & -1 & 1 & -1\end{array}\right]$ & $M(2 B)=\left[\begin{array}{cccccc}1 & 1 & 1 & 1 & 1 & 1 \\
1 & -1 & 1 & -1 & 1 & -1 \\
1 & 1 & 1 & -1 & -1 & -1 \\
6 & 0 & -2 & -6 & 0 & 2 \\
6 & 0 & -2 & 6 & 0 & -2 \\
1 & -1 & 1 & 1 & -1 & 1\end{array}\right]$ \\
\hline$M(3 B)=\left[\begin{array}{cccc}1 & 1 & 1 & 1 \\
3 & 1 & -3 & -1 \\
3 & -1 & 3 & -1 \\
1 & -1 & -1 & 1\end{array}\right]$ & $M(6 A)=\left[\begin{array}{cccc}1 & 1 & 1 & 1 \\
1 & 1 & -1 & -1 \\
1 & -1 & -1 & 1 \\
1 & -1 & 1 & -1\end{array}\right]$ \\
$M(4 B)=\left[\begin{array}{cccccc}1 & 1 & 1 & 1 & 1 & 1 \\
1 & -1 & 1 & 1 & -1 & 1 \\
1 & -1 & -1 & -1 & 1 & 1 \\
2 & 0 & -2 & 2 & 0 & -2 \\
2 & 0 & 2 & -2 & 0 & -2 \\
1 & 1 & -1 & -1 & -1 & 1\end{array}\right]$ & $M(6 B)=\left[\begin{array}{cc}1 & 1 \\
1 & -1\end{array}\right]$ \\
\hline
\end{tabular}

6. The Character TABle of $\bar{G}=2^{5}: \operatorname{GL}(4,2)$

Now, we have:

(1) The conjugacy classes of $\bar{G}=2^{5}: G L(4,2)$ (Table 1);

(2) The character tables of all the inertia factors (by using GAP [18]);

(3) The fusions of conjugacy classes of the inertia factors into classes of GL(4,2) (Table $2 \&$ Table 3);

(4) The Fischer matrices of classes of $\bar{G}=2^{5}: G L(4,2)$ (Table 4);

Thus, we can construct the character table of the $2^{5}: G L(4,2)$ as follows:

Table 5. The Character Table of $\bar{G}=2^{5}: G L(4,2)$

\begin{tabular}{|c|c|c|c|c|c|c|c|c|c|c|}
\hline Class & $1 \mathrm{a}$ & $2 \mathrm{a}$ & $2 \mathrm{~b}$ & $2 \mathrm{c}$ & $3 \mathrm{a}$ & $6 \mathrm{a}$ & $5 \mathrm{a}$ & $10 \mathrm{a}$ & $15 \mathrm{a}$ & $30 \mathrm{a}$ \\
\hline Order & 1 & 2 & 2 & 2 & 3 & 6 & 5 & 10 & 15 & 30 \\
\hline Size & 1 & 1 & 15 & 15 & 179 & 17 & 21504 & 21504 & 21504 & 21504 \\
& & & & & 2 & 92 & & & & \\
\hline $\mathrm{X} 1$ & 1 & 1 & 1 & 1 & 1 & 1 & 1 & 1 & 1 & 1 \\
$\mathrm{X} 2$ & 7 & 7 & 7 & 7 & 4 & 4 & 2 & 2 & -1 & -1 \\
$\mathrm{X} 3$ & 14 & 14 & 14 & 14 & -1 & -1 & -1 & -1 & -1 & -1 \\
$\mathrm{X} 4$ & 20 & 20 & 20 & 20 & 5 & 5 & 0 & 0 & 0 & 0 \\
$\mathrm{X} 5$ & 21 & 21 & 21 & 21 & 6 & 6 & 1 & 1 & 1 & 1 \\
$\mathrm{X} 6$ & 21 & 21 & 21 & 21 & -3 & -3 & 1 & 1 & $\mathrm{~A}$ & $\mathrm{~A}$ \\
$\mathrm{X} 7$ & 21 & 21 & 21 & 21 & -3 & -3 & 1 & 1 & $/ \mathrm{A}$ & $/ \mathrm{A}$ \\
$\mathrm{X} 8$ & 28 & 28 & 28 & 28 & 1 & 1 & -2 & -2 & 1 & 1 \\
$\mathrm{X} 9$ & 35 & 35 & 35 & 35 & 5 & 5 & 0 & 0 & 0 & 0 \\
$\mathrm{X} 10$ & 45 & 45 & 45 & 45 & 0 & 0 & 0 & 0 & 0 & 0 \\
$\mathrm{X} 11$ & 45 & 45 & 45 & 45 & 0 & 0 & 0 & 0 & 0 & 0 \\
$\mathrm{X} 12$ & 56 & 56 & 56 & 56 & -4 & -4 & 1 & 1 & 1 & 1 \\
$\mathrm{X} 13$ & 64 & 64 & 64 & 64 & 4 & 4 & -1 & -1 & -1 & -1 \\
$\mathrm{X} 14$ & 70 & 70 & 70 & 70 & -5 & -5 & 0 & 0 & 0 & 0 \\
$\mathrm{X} 15$ & 15 & 15 & -1 & -1 & 0 & 0 & 0 & 0 & 0 & 0 \\
$\mathrm{X} 16$ & 45 & 45 & -3 & -3 & 0 & 0 & 0 & 0 & 0 & 0 \\
$\mathrm{X} 17$ & 45 & 45 & -3 & -3 & 0 & 0 & 0 & 0 & 0 & 0 \\
$\mathrm{X} 18$ & 90 & 90 & -6 & -6 & 0 & 0 & 0 & 0 & 0 & 0 \\
$\mathrm{X} 19$ & 105 & 105 & -7 & -7 & 0 & 0 & 0 & 0 & 0 & 0 \\
$\mathrm{X} 20$ & 105 & 105 & -7 & -7 & 0 & 0 & 0 & 0 & 0 & 0 \\
$\mathrm{X} 21$ & 105 & 105 & -7 & -7 & 0 & 0 & 0 & 0 & 0 & 0 \\
X22 & 120 & 120 & -8 & -8 & 0 & 0 & 0 & 0 & 0 & 0 \\
X23 & 210 & 210 & -14 & -14 & 0 & 0 & 0 & 0 & 0 & 0 \\
X24 & 315 & 315 & -21 & -21 & 0 & 0 & 0 & 0 & 0 & 0 \\
\hline
\end{tabular}


The Fischer-Cliford Matrices and Character Table of the Group $2^{5}: G L(4,2)$

\begin{tabular}{|c|c|c|c|c|c|c|c|c|c|c|}
\hline $\mathrm{X} 25$ & 315 & 315 & -21 & -21 & 0 & 0 & 0 & 0 & 0 & 0 \\
$\mathrm{X} 26$ & 15 & -15 & 1 & -1 & 0 & 0 & 0 & 0 & 0 & 0 \\
$\mathrm{X} 27$ & 45 & -45 & 3 & -3 & 0 & 0 & 0 & 0 & 0 & 0 \\
$\mathrm{X} 28$ & 45 & -45 & 3 & -3 & 0 & 0 & 0 & 0 & 0 & 0 \\
$\mathrm{X} 29$ & 90 & -90 & 6 & -6 & 0 & 0 & 0 & 0 & 0 & 0 \\
$\mathrm{X} 30$ & 105 & -105 & 7 & -7 & 0 & 0 & 0 & 0 & 0 & 0 \\
$\mathrm{X} 31$ & 105 & -105 & 7 & -7 & 0 & 0 & 0 & 0 & 0 & 0 \\
$\mathrm{X} 32$ & 105 & -105 & 7 & -7 & 0 & 0 & 0 & 0 & 0 & 0 \\
$\mathrm{X} 33$ & 120 & -120 & 8 & -8 & 0 & 0 & 0 & 0 & 0 & 0 \\
$\mathrm{X} 34$ & 210 & -210 & 14 & -14 & 0 & 0 & 0 & 0 & 0 & 0 \\
$\mathrm{X} 35$ & 315 & -315 & 21 & -21 & 0 & 0 & 0 & 0 & 0 & 0 \\
$\mathrm{X} 36$ & 315 & -315 & 21 & -21 & 0 & 0 & 0 & 0 & 0 & 0 \\
$\mathrm{X} 37$ & 1 & -1 & -1 & 1 & 1 & -1 & 1 & -1 & 1 & -1 \\
$\mathrm{X} 38$ & 7 & -7 & -7 & 7 & 4 & -4 & 2 & -2 & -1 & 1 \\
$\mathrm{X} 39$ & 14 & -14 & -14 & 14 & -1 & 1 & -1 & 1 & -1 & 1 \\
$\mathrm{X} 40$ & 20 & -20 & -20 & 20 & 5 & -5 & 0 & 0 & 0 & 0 \\
$\mathrm{X} 41$ & 21 & -21 & -21 & 21 & 6 & -6 & 1 & -1 & 1 & -1 \\
$\mathrm{X} 42$ & 21 & -21 & -21 & 21 & -3 & 3 & 1 & -1 & $/ \mathrm{A}$ & $-/ \mathrm{A}$ \\
$\mathrm{X} 43$ & 21 & -21 & -21 & 21 & -3 & 3 & 1 & -1 & $\mathrm{~A}$ & $-\mathrm{A}$ \\
$\mathrm{X} 44$ & 28 & -28 & -28 & 28 & 1 & -1 & -2 & 2 & 1 & -1 \\
$\mathrm{X} 45$ & 35 & -35 & -35 & 35 & 5 & -5 & 0 & 0 & 0 & 0 \\
$\mathrm{X} 46$ & 45 & -45 & -45 & 45 & 0 & 0 & 0 & 0 & 0 & 0 \\
$\mathrm{X} 47$ & 45 & -45 & -45 & 45 & 0 & 0 & 0 & 0 & 0 & 0 \\
$\mathrm{X} 48$ & 56 & -56 & -56 & 56 & -4 & 4 & -4 & 4 & 1 & -1 \\
$\mathrm{X} 49$ & 64 & -64 & -64 & 64 & 4 & -4 & -1 & 1 & -1 & 1 \\
$\mathrm{X} 50$ & 70 & -70 & -70 & 70 & -5 & 5 & 0 & 0 & 0 & 0 \\
\hline
\end{tabular}

\begin{tabular}{|c|c|c|c|c|c|c|c|c|c|}
\hline Class & $15 \mathrm{~b}$ & $30 \mathrm{~b}$ & $7 a$ & $14 \mathrm{a}$ & $14 \mathrm{~b}$ & $14 \mathrm{c}$ & $7 b$ & $14 \mathrm{~d}$ & $14 \mathrm{e}$ \\
\hline Order & 15 & 30 & 7 & 14 & 14 & 14 & 7 & 14 & 14 \\
\hline Size & 21504 & 21504 & 23040 & 23040 & 23040 & 23040 & 23040 & 23040 & 23040 \\
\hline $\mathrm{X} 1$ & 1 & 1 & 1 & 1 & 1 & 1 & 1 & 1 & 1 \\
\hline $\mathrm{X} 2$ & -1 & -1 & 0 & 0 & 0 & 0 & 0 & 0 & 0 \\
\hline X3 & -1 & -1 & 0 & 0 & 0 & 0 & 0 & 0 & 0 \\
\hline $\mathrm{X} 4$ & 0 & 0 & -1 & -1 & -1 & -1 & -1 & -1 & -1 \\
\hline $\mathrm{X} 5$ & 1 & 1 & 0 & 0 & 0 & 0 & 0 & 0 & 0 \\
\hline X6 & /A & /A & 0 & 0 & 0 & 0 & 0 & 0 & 0 \\
\hline X7 & A & A & 0 & 0 & 0 & 0 & 0 & 0 & 0 \\
\hline $\mathrm{X} 8$ & 1 & 1 & 0 & 0 & 0 & 0 & 0 & 0 & 0 \\
\hline X9 & 0 & 0 & 0 & 0 & 0 & 0 & 0 & 0 & 0 \\
\hline X10 & 0 & 0 & B & B & B & B & /B & /B & /B \\
\hline X11 & 0 & 0 & /B & /B & /B & /B & B & B & B \\
\hline X12 & 1 & 1 & 0 & 0 & 0 & 0 & 0 & 0 & 0 \\
\hline X13 & -1 & -1 & 1 & 1 & 1 & 1 & 1 & 1 & 1 \\
\hline X14 & 0 & 0 & 0 & 0 & 0 & 0 & 0 & 0 & 0 \\
\hline X15 & 0 & 0 & 1 & 1 & -1 & -1 & 1 & 1 & -1 \\
\hline X16 & 0 & 0 & A & A & $-A$ & $-A$ & /A & /A & $-/ A$ \\
\hline X17 & 0 & 0 & /A & /A & $-/ A$ & $-/ A$ & A & A & $-A$ \\
\hline X18 & 0 & 0 & -1 & -1 & 1 & 1 & -1 & -1 & 1 \\
\hline X19 & 0 & 0 & 0 & 0 & 0 & 0 & 0 & 0 & 0 \\
\hline X20 & 0 & 0 & 0 & 0 & 0 & 0 & 0 & 0 & 0 \\
\hline $\mathrm{X} 21$ & 0 & 0 & 0 & 0 & 0 & 0 & 0 & 0 & 0 \\
\hline X22 & 0 & 0 & 1 & 1 & -1 & -1 & 1 & 1 & -1 \\
\hline X23 & 0 & 0 & 0 & 0 & 0 & 0 & 0 & 0 & 0 \\
\hline X24 & 0 & 0 & 0 & 0 & -0 & 0 & 0 & 0 & 0 \\
\hline X25 & 0 & 0 & 0 & 0 & 0 & 0 & 0 & 0 & 0 \\
\hline X26 & 0 & 0 & 1 & -1 & -1 & 1 & 1 & -1 & -1 \\
\hline $\mathrm{X} 27$ & 0 & 0 & A & $-A$ & $-A$ & A & /A & $-/ \mathrm{A}$ & $-/ A$ \\
\hline $\mathrm{X} 28$ & 0 & 0 & /A & $-/ A$ & $-/ \mathrm{A}$ & /A & A & $-A$ & $-A$ \\
\hline X29 & 0 & 0 & -1 & 1 & 1 & -1 & -1 & 1 & 1 \\
\hline X30 & 0 & 0 & 0 & 0 & 0 & 0 & 0 & 0 & 0 \\
\hline X31 & 0 & 0 & 0 & 0 & 0 & 0 & 0 & 0 & 0 \\
\hline X32 & 0 & 0 & 0 & 0 & 0 & 0 & 0 & 0 & 0 \\
\hline X33 & 0 & 0 & 1 & -1 & -1 & 1 & 1 & -1 & -1 \\
\hline X34 & 0 & 0 & 0 & 0 & 0 & 0 & 0 & 0 & 0 \\
\hline X35 & 0 & 0 & 0 & 0 & 0 & 0 & 0 & 0 & 0 \\
\hline X36 & 0 & 0 & 0 & 0 & 0 & 0 & 0 & 0 & 0 \\
\hline X37 & 1 & -1 & 1 & -1 & 1 & -1 & 1 & -1 & 1 \\
\hline X38 & -1 & 1 & 0 & 0 & 0 & 0 & 0 & 0 & 0 \\
\hline X39 & -1 & 1 & 0 & 0 & 0 & 0 & 0 & 0 & 0 \\
\hline
\end{tabular}


The Fischer-Cliford Matrices and Character Table of the Group $2^{5}: G L(4,2)$

\begin{tabular}{|c|c|c|c|c|c|c|c|c|c|}
\hline X40 & 0 & 0 & -1 & 1 & -1 & 1 & -1 & 1 & -1 \\
\hline X41 & 1 & -1 & 0 & 0 & 0 & 0 & 0 & 0 & 0 \\
\hline X42 & A & $-\mathrm{A}$ & 0 & 0 & 0 & 0 & 0 & 0 & 0 \\
\hline X43 & /A & $-/ A$ & 0 & 0 & 0 & 0 & 0 & 0 & 0 \\
\hline X44 & 1 & -1 & 0 & 0 & 0 & 0 & 0 & 0 & 0 \\
\hline X45 & 0 & 0 & 0 & 0 & 0 & 0 & 0 & 0 & 0 \\
\hline X46 & 0 & 0 & B & $-B$ & B & $-B$ & $/ \mathrm{B}$ & $-/ B$ & /B \\
\hline X47 & 0 & 0 & /B & $-/ B$ & $/ \mathrm{B}$ & $-/ B$ & B & $-B$ & B \\
\hline X48 & 1 & -1 & 0 & 0 & 0 & 0 & 0 & 0 & 0 \\
\hline X49 & -1 & 1 & 1 & -1 & 1 & -1 & 1 & -1 & 1 \\
\hline X50 & 0 & 0 & 0 & 0 & 0 & 0 & 0 & 0 & 0 \\
\hline
\end{tabular}

\begin{tabular}{|c|c|c|c|c|c|c|c|c|c|}
\hline Class & $14 \mathrm{f}$ & $2 \mathrm{~d}$ & $2 \mathrm{e}$ & $4 a$ & $4 \mathrm{~b}$ & $4 \mathrm{c}$ & $8 \mathrm{a}$ & $4 \mathrm{~d}$ & $8 \mathrm{~b}$ \\
\hline Order & 14 & 2 & 2 & 4 & 4 & 4 & 8 & 4 & 8 \\
\hline Size & 23040 & 840 & 840 & 2520 & 2520 & 20160 & 20160 & 20160 & 20160 \\
\hline $\mathrm{X} 1$ & 1 & 1 & 1 & 1 & 1 & 1 & 1 & 1 & 1 \\
\hline X2 & 0 & 3 & 3 & 3 & 3 & 1 & 1 & 1 & 1 \\
\hline X3 & 0 & 2 & 2 & 2 & 2 & 0 & 0 & 0 & 0 \\
\hline $\mathrm{X} 4$ & -1 & 4 & 4 & 4 & 4 & 0 & 0 & 0 & 0 \\
\hline X5 & 0 & 1 & 1 & 1 & 1 & -1 & -1 & -1 & -1 \\
\hline X6 & 0 & 1 & 1 & 1 & 1 & -1 & -1 & -1 & -1 \\
\hline X7 & 0 & 1 & 1 & 1 & 1 & -1 & -1 & -1 & -1 \\
\hline X8 & 0 & 4 & 4 & 4 & 4 & 0 & 0 & 0 & 0 \\
\hline X9 & 0 & -5 & -5 & -5 & -5 & -1 & -1 & -1 & -1 \\
\hline X10 & $/ \mathrm{B}$ & -3 & -3 & -3 & -3 & 1 & 1 & 1 & 1 \\
\hline X11 & B & -3 & -3 & -3 & -3 & 1 & 1 & 1 & 1 \\
\hline X12 & 0 & 0 & 0 & 0 & 0 & 0 & 0 & 0 & 0 \\
\hline X13 & 1 & 0 & 0 & 0 & 0 & 0 & 0 & 0 & 0 \\
\hline X14 & 0 & 2 & 2 & 2 & 2 & 0 & 0 & 0 & 0 \\
\hline X15 & -1 & 3 & -3 & -1 & 1 & 1 & 1 & -1 & -1 \\
\hline X16 & -/A & -3 & 3 & 1 & -1 & 1 & 1 & -1 & -1 \\
\hline X17 & $-A$ & -3 & 3 & 1 & -1 & 1 & 1 & -1 & -1 \\
\hline X18 & 1 & 6 & -6 & -2 & 2 & 0 & 0 & 0 & 0 \\
\hline X19 & 0 & -3 & 3 & 1 & -1 & -1 & -1 & 1 & 1 \\
\hline X20 & 0 & -3 & 3 & 1 & -1 & -1 & -1 & 1 & 1 \\
\hline $\mathrm{X} 21$ & 0 & 9 & -9 & -3 & 3 & 1 & 1 & -1 & -1 \\
\hline X22 & -1 & 0 & 0 & 0 & 0 & 0 & 0 & 0 & 0 \\
\hline $\mathrm{X} 23$ & 0 & 6 & -6 & -2 & 2 & 0 & 0 & 0 & 0 \\
\hline X24 & 0 & -9 & 9 & 3 & -3 & 1 & 1 & -1 & -1 \\
\hline X25 & 0 & 3 & -3 & -1 & 1 & -1 & -1 & 1 & 1 \\
\hline X26 & 1 & 3 & 3 & -1 & -1 & 1 & -1 & -1 & 1 \\
\hline $\mathrm{X} 27$ & /A & -3 & -3 & 1 & 1 & 1 & -1 & -1 & 1 \\
\hline X28 & A & -3 & -3 & 1 & 1 & 1 & -1 & -1 & 1 \\
\hline X29 & -1 & 6 & 6 & -2 & -2 & 0 & 0 & 0 & 0 \\
\hline
\end{tabular}


The Fischer-Cliford Matrices and Character Table of the Group $2^{5}: G L(4,2)$

\begin{tabular}{|c|c|c|c|c|c|c|c|c|c|}
\hline $\mathrm{X} 30$ & 0 & -3 & -3 & 1 & 1 & -1 & 1 & 1 & -1 \\
\hline X31 & 0 & -3 & -3 & 1 & 1 & -1 & 1 & 1 & -1 \\
\hline X32 & 0 & 9 & 9 & -3 & -3 & 1 & -1 & -1 & 1 \\
\hline X33 & 1 & 0 & 0 & 0 & 0 & 0 & 0 & 0 & 0 \\
\hline X34 & 0 & 6 & 6 & -2 & -2 & 0 & 0 & 0 & 0 \\
\hline X35 & 0 & -9 & -9 & 3 & 3 & 1 & -1 & -1 & 1 \\
\hline X36 & 0 & 3 & 3 & -1 & -1 & -1 & 1 & 1 & -1 \\
\hline X37 & -1 & 1 & -1 & 1 & -1 & 1 & -1 & 1 & -1 \\
\hline X38 & 0 & 3 & -3 & 3 & -3 & 1 & -1 & 1 & -1 \\
\hline X39 & 0 & 2 & -2 & 2 & -2 & 0 & 0 & 0 & 0 \\
\hline $\mathrm{X} 40$ & 1 & 4 & -4 & 4 & -4 & 0 & 0 & 0 & 0 \\
\hline X41 & 0 & 1 & -1 & 1 & -1 & -1 & 1 & -1 & 1 \\
\hline $\mathrm{X} 42$ & 0 & 1 & -1 & 1 & -1 & -1 & 1 & -1 & 1 \\
\hline $\mathrm{X} 43$ & 0 & 1 & -1 & 1 & -1 & -1 & 1 & -1 & 1 \\
\hline X44 & 0 & 4 & -4 & 4 & -4 & 0 & 0 & 0 & 0 \\
\hline $\mathrm{X} 45$ & 0 & -5 & 5 & -5 & 5 & -1 & 1 & -1 & 1 \\
\hline X46 & $-/ B$ & -3 & 3 & -3 & 3 & 1 & -1 & 1 & -1 \\
\hline X47 & $-B$ & -3 & 3 & -3 & 3 & 1 & -1 & 1 & -1 \\
\hline X48 & 0 & 0 & 0 & 0 & 0 & 0 & 0 & 0 & 0 \\
\hline X49 & -1 & 0 & 0 & 0 & 0 & 0 & 0 & 0 & 0 \\
\hline X50 & 0 & 2 & -2 & 2 & -2 & 0 & 0 & 0 & 0 \\
\hline
\end{tabular}

\begin{tabular}{|c|c|c|c|c|c|c|c|c|c|c|}
\hline Class & $2 \mathrm{f}$ & $4 \mathrm{e}$ & $2 g$ & $2 \mathrm{~h}$ & $4 \mathrm{f}$ & $2 \mathrm{i}$ & $3 b$ & $6 \mathrm{~b}$ & $6 \mathrm{c}$ & $6 \mathrm{~d}$ \\
\hline Order & 2 & 4 & 2 & 2 & 4 & 2 & 3 & 6 & 6 & 6 \\
\hline Size & 210 & 840 & 630 & 210 & 840 & 630 & 4480 & 13440 & 4480 & 13440 \\
\hline $\mathrm{X} 1$ & 1 & 1 & 1 & 1 & 1 & 1 & 1 & 1 & 1 & 1 \\
\hline $\mathrm{X} 2$ & -1 & -1 & -1 & -1 & -1 & -1 & 1 & 1 & 1 & 1 \\
\hline X3 & 6 & 6 & 6 & 6 & 6 & 6 & 2 & 2 & 2 & 2 \\
\hline $\mathrm{X} 4$ & 4 & 4 & 4 & 4 & 4 & 4 & -1 & -1 & -1 & -1 \\
\hline X5 & -3 & -3 & -3 & -3 & -3 & -3 & 0 & 0 & 0 & 0 \\
\hline X6 & -3 & -3 & -3 & -3 & -3 & -3 & 0 & 0 & 0 & 0 \\
\hline X7 & -3 & -3 & -3 & -3 & -3 & -3 & 0 & 0 & 0 & 0 \\
\hline X8 & -4 & -4 & -4 & -4 & -4 & -4 & 1 & 1 & 1 & 1 \\
\hline X9 & 3 & 3 & 3 & 3 & 3 & 3 & 2 & 2 & 2 & 2 \\
\hline X10 & -3 & -3 & -3 & -3 & -3 & -3 & 0 & 0 & 0 & 0 \\
\hline X11 & -3 & -3 & -3 & -3 & -3 & -3 & 0 & 0 & 0 & 0 \\
\hline $\mathrm{X} 12$ & 8 & 8 & 8 & 8 & 8 & 8 & -1 & -1 & -1 & -1 \\
\hline X13 & 0 & 0 & 0 & 0 & 0 & 0 & -2 & -2 & -2 & -2 \\
\hline X14 & -2 & -2 & -2 & -2 & -2 & -2 & 1 & 1 & 1 & 1 \\
\hline
\end{tabular}


The Fischer-Cliford Matrices and Character Table of the Group $2^{5}: G L(4,2)$

\begin{tabular}{|c|c|c|c|c|c|c|c|c|c|c|}
\hline $\mathrm{X} 15$ & 7 & -1 & -1 & -7 & 1 & 1 & 3 & 1 & -3 & -1 \\
\hline X16 & -3 & -3 & 5 & 3 & 3 & -5 & 0 & 0 & 0 & 0 \\
\hline X17 & -3 & -3 & 5 & 3 & 3 & -5 & 0 & 0 & 0 & 0 \\
\hline $\mathrm{X} 18$ & 18 & -6 & 2 & -18 & 6 & -2 & 0 & 0 & 0 & 0 \\
\hline X19 & 17 & 1 & -7 & -17 & -1 & 7 & 3 & 1 & -3 & -1 \\
\hline $\mathrm{X} 20$ & 1 & -7 & 9 & -1 & 7 & -9 & 3 & 1 & -3 & -1 \\
\hline $\mathrm{X} 21$ & -7 & 1 & 1 & 7 & -1 & -1 & 3 & 1 & -3 & -1 \\
\hline $\mathrm{X} 22$ & 8 & -8 & 8 & -8 & 8 & -8 & -3 & -1 & 3 & 1 \\
\hline $\mathrm{X} 23$ & 10 & 2 & -6 & -10 & -2 & 6 & -3 & -1 & 3 & 1 \\
\hline X24 & 3 & 3 & -5 & -3 & -3 & 5 & 0 & 0 & 0 & 0 \\
\hline $\mathrm{X} 25$ & -21 & 3 & 3 & 21 & -3 & -3 & 0 & 0 & 0 & 0 \\
\hline X26 & 7 & 1 & -1 & 5 & -1 & -3 & 3 & -1 & 3 & -1 \\
\hline X27 & -3 & 3 & 5 & -9 & -3 & -1 & 0 & 0 & 0 & 0 \\
\hline $\mathrm{X} 28$ & -3 & 3 & 5 & -9 & -3 & -1 & 0 & 0 & 0 & 0 \\
\hline X29 & 18 & 6 & 2 & 6 & -6 & -10 & 0 & 0 & 0 & 0 \\
\hline X30 & 17 & -1 & -7 & 19 & 1 & -5 & 3 & -1 & 3 & -1 \\
\hline X31 & 1 & 7 & 9 & -13 & -7 & -5 & 3 & -1 & 3 & -1 \\
\hline X32 & -7 & -1 & 1 & -5 & 1 & 3 & 3 & -1 & 3 & -1 \\
\hline X33 & 8 & 8 & 8 & -8 & -8 & -8 & -3 & 1 & -3 & 1 \\
\hline X34 & 10 & -2 & -6 & 14 & 2 & -2 & -3 & 1 & -3 & 1 \\
\hline X35 & 3 & -3 & -5 & 9 & 3 & 1 & 0 & 0 & 0 & 0 \\
\hline X36 & -21 & -3 & 3 & -15 & 3 & 9 & 0 & 0 & 0 & 0 \\
\hline X37 & 1 & -1 & 1 & 1 & -1 & 1 & 1 & -1 & -1 & 1 \\
\hline X38 & -1 & 1 & -1 & -1 & 1 & -1 & 1 & -1 & -1 & 1 \\
\hline X39 & 6 & -6 & 6 & 6 & -6 & 6 & 2 & -2 & -2 & 2 \\
\hline $\mathrm{X} 40$ & 4 & -4 & 4 & 4 & -4 & 4 & -1 & 1 & 1 & -1 \\
\hline X41 & -3 & 3 & -3 & -3 & 3 & -3 & 0 & 0 & 0 & 0 \\
\hline $\mathrm{X} 42$ & -3 & 3 & -3 & -3 & 3 & -3 & 0 & 0 & 0 & 0 \\
\hline $\mathrm{X} 43$ & -3 & 3 & -3 & -3 & 3 & -3 & 0 & 0 & 0 & 0 \\
\hline X44 & -4 & 4 & -4 & -4 & 4 & -4 & 1 & -1 & -1 & 1 \\
\hline $\mathrm{X} 45$ & 3 & -3 & 3 & 3 & -3 & 3 & 2 & -2 & -2 & 2 \\
\hline X46 & -3 & 3 & -3 & -3 & 3 & -3 & 0 & 0 & 0 & 0 \\
\hline $\mathrm{X} 47$ & -3 & 3 & -3 & -3 & 3 & -3 & 0 & 0 & 0 & 0 \\
\hline $\mathrm{X} 48$ & 8 & -8 & 8 & 8 & -8 & 8 & -1 & 1 & 1 & -1 \\
\hline X49 & 0 & 0 & 0 & 0 & 0 & 0 & -2 & 2 & 2 & -2 \\
\hline X50 & -2 & 2 & -2 & -2 & 2 & -2 & 1 & -1 & -1 & 1 \\
\hline
\end{tabular}


The Fischer-Cliford Matrices and Character Table of the Group $2^{5}: G L(4,2)$

\begin{tabular}{|c|c|c|c|c|c|c|c|c|c|}
\hline Class & $6 \mathrm{e}$ & $12 \mathrm{a}$ & $6 \mathrm{f}$ & $12 \mathrm{~b}$ & $4 \mathrm{~g}$ & $4 \mathrm{~h}$ & $4 \mathrm{i}$ & $4 \mathrm{j}$ & $4 \mathrm{k}$ \\
\hline Order & 6 & 12 & 6 & 12 & 4 & 4 & 4 & 4 & 4 \\
\hline Size & 26880 & 26880 & 26880 & 26880 & 5040 & 10080 & 5040 & 5040 & 10080 \\
\hline $\mathrm{X} 1$ & 1 & 1 & 1 & 1 & 1 & 1 & 1 & 1 & 1 \\
\hline $\mathrm{X} 2$ & -1 & -1 & -1 & -1 & -1 & -1 & -1 & -1 & -1 \\
\hline $\mathrm{X} 3$ & 0 & 0 & 0 & 0 & 2 & 2 & 2 & 2 & 2 \\
\hline $\mathrm{X} 4$ & 1 & 1 & 1 & 1 & 0 & 0 & 0 & 0 & 0 \\
\hline X5 & 0 & 0 & 0 & 0 & 1 & 1 & 1 & 1 & 1 \\
\hline X6 & 0 & 0 & 0 & 0 & 1 & 1 & 1 & 1 & 1 \\
\hline $\mathrm{X} 7$ & 0 & 0 & 0 & 0 & 1 & 1 & 1 & 1 & 1 \\
\hline $\mathrm{X} 8$ & -1 & -1 & -1 & -1 & 0 & 0 & 0 & 0 & 0 \\
\hline $\mathrm{X} 9$ & 0 & 0 & 0 & 0 & -1 & -1 & -1 & -1 & -1 \\
\hline $\mathrm{X} 10$ & 0 & 0 & 0 & 0 & 1 & 1 & 1 & 1 & 1 \\
\hline X11 & 0 & 0 & 0 & 0 & 1 & 1 & 1 & 1 & 1 \\
\hline $\mathrm{X} 12$ & -1 & -1 & -1 & -1 & 0 & 0 & 0 & 0 & 0 \\
\hline $\mathrm{X} 13$ & 0 & 0 & 0 & 0 & 0 & 0 & 0 & 0 & 0 \\
\hline X14 & 1 & 1 & 1 & 1 & -2 & -2 & -2 & -2 & -2 \\
\hline X15 & 1 & 1 & -1 & -1 & 3 & -1 & -1 & 3 & -1 \\
\hline X16 & 0 & 0 & 0 & 0 & 1 & 1 & -3 & 1 & 1 \\
\hline X17 & 0 & 0 & 0 & 0 & 1 & 1 & -3 & 1 & 1 \\
\hline $\mathrm{X} 18$ & 0 & 0 & 0 & 0 & 2 & -2 & 2 & 2 & -2 \\
\hline X19 & -1 & -1 & 1 & 1 & 1 & 1 & -3 & 1 & 1 \\
\hline $\mathrm{X} 20$ & 1 & 1 & -1 & -1 & -3 & 1 & 1 & -3 & 1 \\
\hline $\mathrm{X} 21$ & -1 & -1 & 1 & 1 & -3 & 1 & 1 & -3 & 1 \\
\hline X22 & -1 & -1 & 1 & 1 & 0 & 0 & 0 & 0 & 0 \\
\hline $\mathrm{X} 23$ & 1 & 1 & -1 & -1 & -2 & 2 & -2 & -2 & 2 \\
\hline $\mathrm{X} 24$ & 0 & 0 & 0 & 0 & -1 & -1 & 3 & -1 & -1 \\
\hline $\mathrm{X} 25$ & 0 & 0 & 0 & 0 & 3 & -1 & -1 & 3 & -1 \\
\hline $\mathrm{X} 26$ & 1 & -1 & -1 & 1 & 3 & -1 & 1 & -3 & 1 \\
\hline $\mathrm{X} 27$ & 0 & 0 & 0 & 0 & 1 & 1 & 3 & -1 & -1 \\
\hline $\mathrm{X} 28$ & 0 & 0 & 0 & 0 & 1 & 1 & 3 & -1 & -1 \\
\hline X29 & 0 & 0 & 0 & 0 & 2 & -2 & -2 & -2 & 2 \\
\hline X30 & -1 & 1 & 1 & -1 & 1 & 1 & 3 & -1 & -1 \\
\hline X31 & 1 & -1 & -1 & 1 & -3 & 1 & -1 & 3 & -1 \\
\hline X32 & -1 & 1 & 1 & -1 & -3 & 1 & -1 & 3 & -1 \\
\hline X33 & -1 & 1 & 1 & -1 & 0 & 0 & 0 & 0 & 0 \\
\hline X34 & 1 & -1 & -1 & 1 & -2 & 2 & 2 & 2 & -2 \\
\hline X35 & 0 & 0 & 0 & 0 & -1 & -1 & -3 & 1 & 1 \\
\hline X36 & 0 & 0 & 0 & 0 & 3 & -1 & 1 & -3 & 1 \\
\hline
\end{tabular}


The Fischer-Cliford Matrices and Character Table of the Group $2^{5}: G L(4,2)$

\begin{tabular}{|c|c|c|c|c|c|c|c|c|c|}
\hline X37 & 1 & -1 & 1 & -1 & 1 & 1 & -1 & -1 & -1 \\
\hline X38 & -1 & 1 & -1 & 1 & -1 & -1 & 1 & 1 & 1 \\
\hline X39 & 0 & 0 & 0 & 0 & 2 & 2 & -2 & -2 & -2 \\
\hline $\mathrm{X} 40$ & 1 & -1 & 1 & -1 & 0 & 0 & 0 & 0 & 0 \\
\hline X41 & 0 & 0 & 0 & 0 & 1 & 1 & -1 & -1 & -1 \\
\hline $\mathrm{X} 42$ & 0 & 0 & 0 & 0 & 1 & 1 & -1 & -1 & -1 \\
\hline $\mathrm{X} 43$ & 0 & 0 & 0 & 0 & 1 & 1 & -1 & -1 & -1 \\
\hline X44 & -1 & 1 & -1 & 1 & 0 & 0 & 0 & 0 & 0 \\
\hline X45 & 0 & 0 & 0 & 0 & -1 & -1 & 1 & 1 & 1 \\
\hline X46 & 0 & 0 & 0 & 0 & 1 & 1 & -1 & -1 & -1 \\
\hline X47 & 0 & 0 & 0 & 0 & 1 & 1 & -1 & -1 & -1 \\
\hline X48 & -1 & 1 & -1 & 1 & 0 & 0 & 0 & 0 & 0 \\
\hline X49 & 0 & 0 & 0 & 0 & 0 & 0 & 0 & 0 & 0 \\
\hline X50 & 1 & -1 & 1 & -1 & -2 & -2 & 2 & 2 & 2 \\
\hline
\end{tabular}

\begin{tabular}{|c|c|c|c|}
\hline Class & 41 & $6 \mathrm{~g}$ & $6 \mathrm{~h}$ \\
\hline Order & 4 & 6 & 6 \\
\hline Size & 5040 & 26880 & 26880 \\
\hline X1 & 1 & 1 & 1 \\
X2 & -1 & 0 & 0 \\
X3 X4 & 2 & -1 & -1 \\
X5 & 0 & 1 & 1 \\
X6 & 1 & -2 & -2 \\
X7 & 1 & 1 & 1 \\
X8 & 0 & 1 & 1 \\
X9 & -1 & 1 & 1 \\
X10 & 1 & 0 & 0 \\
X11 & 1 & 0 & 0 \\
X12 & 0 & 0 & 0 \\
X13 & 0 & 0 & 0 \\
X14 & -2 & -1 & -1 \\
X15 & -1 & 0 & 0 \\
X16 & -3 & 0 & 0 \\
X17 & -3 & 0 \\
X18 & 2 & 0 \\
X19 & -3 & 0 \\
X20 & 1 & 0 \\
\hline
\end{tabular}




\begin{tabular}{|c|c|c|c|}
\hline $\mathrm{X} 21$ & 1 & 0 & 0 \\
\hline X22 & 0 & 0 & 0 \\
\hline X23 & -2 & 0 & 0 \\
\hline X24 & 3 & 0 & 0 \\
\hline X25 & -1 & 0 & 0 \\
\hline $\mathrm{X} 26$ & -1 & 0 & 0 \\
\hline X27 & -3 & 0 & 0 \\
\hline $\mathrm{X} 28$ & -3 & 0 & 0 \\
\hline X29 & 2 & 0 & 0 \\
\hline X30 & -3 & 0 & 0 \\
\hline X31 & 1 & 0 & 0 \\
\hline X32 & 1 & 0 & 0 \\
\hline X33 & 0 & 0 & 0 \\
\hline X34 & -2 & 0 & 0 \\
\hline X35 & 3 & 0 & 0 \\
\hline X36 & -1 & 0 & 0 \\
\hline X37 & 1 & 1 & -1 \\
\hline X38 & -1 & 0 & 0 \\
\hline X39 & 2 & -1 & 1 \\
\hline $\mathrm{X} 40$ & 0 & 1 & -1 \\
\hline $\mathrm{X} 41$ & 1 & -2 & 2 \\
\hline $\mathrm{X} 42$ & 1 & 1 & -1 \\
\hline $\mathrm{X} 43$ & 1 & 1 & -1 \\
\hline X44 & 0 & 1 & -1 \\
\hline $\mathrm{X} 45$ & -1 & 1 & -1 \\
\hline X46 & 1 & 0 & 0 \\
\hline $\mathrm{X} 47$ & 1 & 0 & 0 \\
\hline $\mathrm{X} 48$ & 0 & 0 & 0 \\
\hline X49 & 0 & 0 & 0 \\
\hline X50 & -2 & -1 & 1 \\
\hline
\end{tabular}

Explanation of Character Value Symbols:

$A=\frac{-1-\sqrt{-15}}{2}, \quad B=\frac{-1-\sqrt{-7}}{2}$

\section{REFERENCES}

Clifford A. H., "Representations induced in an invariant subgroup" Ann. of Math., pp. 533 - 550, (1973).

Fischer B., "Clifford matrices" Representation theory of finite groups and finite-dimensional Lie algebras, pp. 1 $-16,(1991)$.

Prins A. L.,"'The Fischer-Clifford Matrices And Character Table of The maximal Subgroup 29: $\left(L_{3}(4): S_{3}\right)$ Of $U_{6}(2): S_{3}$ “, Bull. Iranian Math. Soc., Vol. 42, No. 5, pp. 1179-1195, (2016). 
Prins A. L. and Fray R. L., "The Fischer-Clifford Matrices Of The Inertia Group $2^{7}: O_{6}^{-}(2)$ Of A Maximal Subgroup $2^{7}: S p_{6}$ (2) In $S p_{8}$ (2)", International Journal of Group Theory, Vol.2 No.3 , pp. 19-38, (2013).

Prins A. L. and Fray R. L., "The Fischer-Clifford Matrices Of An Extension Group Of The form $2^{7}:\left(2^{5}: S_{6}\right)$ ", International Journal of Group Theory, Vol. 3 No.2, pp. 21-39, (2014).

Basheer B. M., "Clifford-Fischer Theory Applied to Certain Groups Associated with Symplectic Unitary and Thompson Groups" PhD Thesis, University of KwaZulu-Natal, Pietermaitzburg, (2012).

Basheer B. M., '’n A Group of The Form $2^{14}: S p(6,2)$ ”, QuaestionesMathematicae, pp 1-13, (2015).

Ali F., "Fischer-Clifford Theory for Split and Non-Split Group Extensions" PhD Thesis, University of Natal, Pietermaritzburg, (2001).

Ali F., "The Fischer-Clifford Matrices And Chracter Table Of The Splite Extension $2^{6}: S_{8}$ ", Hacettepe Journal of Mathematics and Statistics, Vol. 43 No.2, pp. 153-171, (2014).

Moori J., "On the Groups G+ and G of the form 210:M22 and 210:M22", PhD Thesis, University of Birmingham, (1975).

Moori J. and SeretloT.,'On An Inertia Factor Group Of $2^{8}: O_{8}^{+}(2)$ ”, Italian Journal of Pure and Applied Mathematics No. 33, pp. 241-254, (2014).

Moori J. and SeretloT.,'On a Maximal Subgroup Of $O_{10}^{+}(2)$ ”, Southeast Asian Bulletin of Mathematics Vol. 39 , pp. 249-263, (2015).

R.L. Fray and A.L. Prins,"A Group Of The Form $2^{7}: S_{8}$ As An Inertia Group Of The Affine Subgroup $2^{7}: S p_{6}(2)$ Of $S p_{8}(2) "$, Internaional Journal Of Modern Mathematical Scince, Vol.13 No.3 (2015), pp.330351 .

Seretlo T., "Fischer-Clifford Matrices and Character Tables of Certain Groups Associated with Simple Groups O+8 (2), HS and Ly", PhD Thesis, University of KwaZulu-Natal, Pietermaitzburg, (2012).

Mpono Z. E., "Fischer Clifford Theory and Character Tables of Group Extensions", PhD Thesis, University of Natal, Pietermaritzburg, (1998).

Maxima, A Computer Algebra System. Version 5.18.1; 2009. Available: http://maxima.sourceforge.net.

Magma program, version 2.20-9. Available: http://magma.maths.usyd.edu.au/magma/.

The GAP Group, GAP - Groups, Algorithms, and Programming, Version 4.8.6; 2016. Available: http://www.gap-system.org.

Wilson R. A. et al.," Atlas of finite group representations”. Available: http://brauer.maths.qmul.ac.uk/Atlas/v3. Gallagher P. X.,” Group characters and normal Hall subgroups”, Nagoya Math. J. 21, pp. 223 - 230, (1962).

Citation: R. I. Elkhatib, "The Fischer-Cliford Matrices and Character Table of the Group 25:GL(4,2) ", International Journal of Scientific and Innovative Mathematical Research, vol. 6, no. 3, p. 11-24, 2018., http://dx.doi.org/10.20431/2347-3142.0603002

Copyright: (C) 2018 Authors. This is an open-access article distributed under the terms of the Creative Commons Attribution License, which permits unrestricted use, distribution, and reproduction in any medium, provided the original author and source are credited. 